\title{
Prognoserelevanz von Body-Mass-Index und Rash für Patienten mit metastasiertem nichtkleinzelligem Lungenkarzinom unter Therapie mit Erlotinib Eine retrospektive-geschlechtsspezifische Analyse
}

\author{
Prognostic Relevance of Body Mass Index and Rash for Patients with Metastatic Non-Small-Cell Lung Cancer \\ under Therapy with Erlotinib \\ A Retrospective, Gender-Specific Analysis
}

Autoren

Institut
B. Böker, H. Lüders, C. Grohé

Klinik für Pneumologie, Evangelische Lungenklinik Berlin-Buch eingereicht 20.7.2011 akzeptiert nach Revision 5. 12.2011

\section{Bibliografie}

Dol http://dx.doi.org/ 10.1055/s-0031-1291617 Pneumologie 2012; 66: 89-95 (c) Georg Thieme Verlag KG Stuttgart · New York ISSN 0934-8387

\section{Korrespondenzadresse Prof. Dr. Christian Grohé Klinik für Pneumologie Evangelische Lungenklinik Berlin-Buch \\ Lindenberger Weg 27 \\ 13125 Berlin \\ christian.grohe@elk-berlin.de}

\section{Zusammenfassung \\ $\nabla$}

Die Therapie mit Tyrosinkinasehemmern der EGFR Signalkaskade, wie z. B. Erlotinib bei Patienten mit nichtkleinzelligen Lungenkarzinomen im Stadium IV, gewinnt zunehmend an Bedeutung. Die Histologie ist ein bekannter Prognosefaktor für das Ansprechen auf diese Therapie, ebenso der EGF-Rezeptor-Mutationsstatus. Weitere Indikatoren, die mit der Prognose assoziiert sind, sind ungenügend definiert, insbesondere fehlen Angaben zur Rolle des Ernährungszustands des Patienten bei einer standardisierten Chemotherapiedosierung der Substanzgruppe. In der vorliegenden retrospektiven Analyse von 275 Patienten, die mit Erlotinib behandelt wurden, wurden weitere klinisch-prognostische Faktoren definiert. Eine stark ausgeprägte Hautreaktion (Rash) war insbesondere bei männlichen Patienten mit einer schlechten Prognose bezüglich des Gesamtüberlebens verbunden. Aktive Raucher entwickeln ebenfalls häufiger intensiven Rash als Nichtraucher und haben auch eine schlechtere Prognose. Patienten mit Untergewicht $(\mathrm{BMI}<18)$ oder starkem Übergewicht (BMI>30) entwickeln nicht häufiger starken Rash als normalgewichtige Patienten, haben unter Erlotinib jedoch auch ein schlechtes Gesamtüberleben. Klinisch relevante Prognosefaktoren wie z.B. der Ernährungszustand sollen in der Zukunft eine größere Rolle in der palliativen Therapie von Patienten mit NSCLC spielen.

\section{Einleitung}

\section{$\nabla$}

Das Lungenkarzinom ist einer der häufigsten soliden Tumoren in den westlichen Industrieländern [1]. In Deutschland und Österreich ist es bei Männern nach dem Prostatakarzinom die zweithäufigste maligne Erkrankung, bei Frauen nach Mammakarzinom und kolorektalem Karzinom die dritthäufigste. Seit über 20 Jahren ist die Inzidenz

\section{Abstract \\ $\nabla$}

Tyrosine kinase inhibition (TKI) such as erlotinib is a well established treatment option in the palliative care of patients with non small cell lung cancer (NSCLC). Histology and sex have been associated with different prognostic outcome measures in patients treated with erlotinib. Furthermore, the degree of rash, developed during treatment might be a relevant marker in respect to tumor response. To dissect these clinical relevant features we analysed a cohort of 275 patients treated with erlotinib in different lines of chemotherapy in our hospital. Nutrition status plays an important role in the prognosis of patients in a palliative chemotherapeutic setting, we therefore included body mass index measurements (BMI) in our analysis. We found that BMI and smoking status influence different survival patterns. Male patients have a poorer survival based on low BMI, rash development and smoking status. We therefore conclude that both nutritional and smoking status should be taken into account in the surveillance of patients with NSCLC in a palliative therapeutic setting under TKI treatment.

bei Männern rückläufig, während sie bei Frauen kontinuierlich ansteigt [1].

Etwa 75 bis 85\% der Lungenkarzinome zählen zu den nichtkleinzelligen Karzinomen (NSCLC), der verbleibende Rest zu den kleinzelligen Karzinomen (SCLC) [2], deren Therapie und Prognose sich grundlegend von erstgenannten unterscheidet. 
Etwa 70\% der Patienten mit Lungenkarzinom haben zum Zeitpunkt der Diagnosestellung ein fortgeschrittenes oder metastasiertes Stadium (Stadium IIIb oder IV), sodass der palliativen und symptomorientierten Therapie bei dieser Erkrankung ein besonderer Stellenwert zukommt.

Die Prognose der Patienten mit Lungenkarzinomen, unabhängig vom Stadium der Erkrankung bei Diagnosestellung und Therapie, ist noch immer schlecht: Die Gesamt-5-Jahres-Überlebensrate liegt bei 15-18\%. Im frühesten Stadium der Erkrankung (Stadium I, Tumorgröße unter $3 \mathrm{~cm}$ ) und operativer Therapie mit R0-Resektion liegt die 5-Jahres-Überlebensrate bei 85\% [11], bereits im Stadium IIa liegt diese nur noch bei 55\%.

In den letzten zehn Jahren wurden einige Studien publiziert, nach deren Ergebnissen sich die mittlere Überlebenszeit der Patienten im Stadium IV verlängert hat (von median 7,9 Monaten auf 11,3 Monate [3, 4]). Diese Beobachtung ist neben den verbesserten supportiven Maßnahmen auch auf die Einführung neuer Substanzen in die Therapie zurückzuführen. So konnte 2008 gezeigt werden, dass durch die Gabe von Pemetrexed zusammen mit Cisplatin in der Erstlinientherapie ein deutlicher Überlebensvorteil für die Patienten mit NSCLC vom Typ Adenokarzinom entsteht [5].

Ende 2005 wurde mit Erlotinib (Tarceva ${ }^{\circledR}$ ) in Deutschland der erste EGFR-Tyrosinkinasehemmer (TKI) für die Therapie des fortgeschrittenen Lungenkarzinoms zugelassen [6]. Ab der Zweitlinientherapie stellte Erlotinib eine Alternative zur Chemotherapie dar, insbesondere bei Patienten in reduziertem Zustand und mit eingeschränkter Knochenmarkreserve. Es konnte nicht nur eine Verlängerung der medianen Überlebenszeit von 4,7 auf 6,7 Monate [6], sondern auch eine Verbesserung der tumorbedingten Symptome (Husten, Dyspnoe, Schmerzen) und der Lebensqualität gezeigt werden [7]. Am meisten profitierten die weiblichen Patienten mit Adenokarzinom, Nichtraucher und Patienten asiatischen Ursprungs. Eine Prognoserelevanz des EGF-Rezeptorstatus konnte von Shepherd et al. zum damaligen Zeitpunkt (noch) nicht gezeigt werden [6].

Seit Juli 2009 ist auch Gefitinib (Iressa ${ }^{\circledR}$ ) für nichtkleinzellige Lungenkarzinome ab der ersten Therapielinie zugelassen, sofern der Tumor eine aktivierende Mutation des EGF-Rezeptors aufweist $[8,9,10]$. Bei dieser Patienten-Subgruppe konnte ein signifikant verlängertes progressionsfreies Überleben im Vergleich zu den mit einer Standard-Chemotherapie behandelten Patienten gezeigt werden (9,5 bzw. 10,4 Monate versus 6,3 bzw. 5,5 Monate) $[9,10]$. Die Ergebnisse der mit asiatischen Patienten durchgeführten Studie, die zur Zulassung von Gefitinib führte, lassen sich auf Patienten anderer Ethnien übertragen, sofern eine aktivierende EGFR-Mutation nachgewiesen wurde. Auch hier waren eine gute Verträglichkeit der Therapie und die wenig beeinträchtigte Lebensqualität hervorzuheben.

Die Wirkungsweise der TKI beruht auf einer reversiblen Hemmung von Anteilen des epidermal growth factor receptors (EGFRRezeptor), einem Glykoprotein, das in $80 \%$ der nichtkleinzelligen Lungenkarzinome exprimiert wird. Die Aktivierung des Rezeptors führt in den Tumorzellen zur Förderung von Wachstum, Angiogenese und Metastasierung.

Seit 2004 ist bekannt, dass bestimmte Mutationen des EGF-Rezeptors zu einer stark erhöhten Sensitivität und damit besseren Ansprechen gegenüber TKI's führt. So zeigte sich, dass eine Deletion im Exon 19 (45-50\% der nachgewiesenen Mutationen), Exon 21 (35-40\%), Exon 18 (3\%) und Exon 20 (3\%) mit einer guten Ansprechrate vergesellschaftet ist, wobei die Patienten mit einer Mutation im Exon 19 noch einmal eine bessere Prognose zu haben scheinen als Patienten mit anderen Mutationen.

Die hauptsächlichen Nebenwirkungen der TKI's sind Hauteffloreszenzen im Sinne einer Rötung (Rash) der Haut, in schwereren Fällen sind Pusteln und eitrige Superinfektionen zu beobachten. Diese Veränderungen zeigen sich am stärksten im Gesicht, am Rücken und im Dekolleté, eine starke Austrocknung der Haut betrifft das gesamte Integument. Bei längerer Anwendungsdauer kann es zu Nagelwallentzündungen (Paronychien) kommen.

Hautnebenwirkungen treten insgesamt bei etwa 75\% der Patienten unter Erlotinib auf, wobei nur 5,4\% dieser Patienten drittoder viertgradige Hautveränderungen haben [23].

Diarrhoen treten als Nebenwirkung von Erlotinib deutlich seltener auf, schwere Diarrhoen (CTC Grad 3) werden in den Studien in einer Häufigkeit von 1,6 bis 6\% angegeben.

Anders als bei anderen tumorspezifischen Therapien ist die Dosis der TKI's bisher unabhängig von Größe und Gewicht gewählt worden. Für Erlotinib liegt die Initialdosis bei $150 \mathrm{mg}$ täglich, für Gefitinib bei $250 \mathrm{mg}$ täglich. Eine Dosisreduktion aufgrund von Nebenwirkungen ist für Gefitinib nicht vorgesehen, für Erlotinib können die Dosisstufen 100 und 50 mg eingesetzt werden.

Neben dem Mutationsstatus des EGF-Rezeptors, dem die größte prognostische Bedeutung zukommt, wurde versucht, noch andere prognostische Faktoren zu definieren. Bisher ist bekannt, dass die Histologie (Adenokarzinom), weibliches Geschlecht, Nichtraucherstatus und asiatische Herkunft prognostisch günstige Faktoren darstellen. Da auch Patienten mit sog. genetischem Wildtyp oder Patienten mit Plattenepithelkarzinomen unter den Langzeitüberlebenden unter Erlotinib sind, wurde versucht, noch andere Faktoren herauszufiltern.

Die Intensität der Hautveränderungen unter Erlotinib wurden als früher Wirksamkeitsparameter [6], aber auch als positiv prognostischer Faktor für das Gesamtüberleben gewertet [2]. Hierbei ist die Beurteilung des Ausprägungsgrades jedoch stark untersucherabhängig.

Die Bedeutung des Ernährungsstatus für die Prognose der Patienten unter Erlotinib wurde bisher noch nicht untersucht. Dass Mangelernährung bei Karzinompatienten zum Zeitpunkt des Therapiebeginns eine schlechte Prognose und schlechtere Lebensqualität zur Folge hat, ist gut bekannt [25]. Der Einfluss starker Übergewichtigkeit auf die Prognose und das Therapieansprechen bei Karzinompatienten wurde jedoch noch nie untersucht. Auch Daten zum Zusammenhang von Body-Mass-Index und Wirksamkeit von Tyrosinkinasehemmern unter identischer Dosierung oder zur Nebenwirkungsrate fehlen bisher.

Dass Nichtraucher mit fortgeschrittenen Lungenkarzinomen eine bessere Prognose und besseres Therapieansprechen (unabhängig vom Therapieregime) haben als Ex-Raucher oder Raucher ist aus großen Untersuchungen bekannt [26]. Auch für Erlotinib wurde bereits frühzeitig bekannt, dass Nie-Raucher ein besseres Therapieansprechen zeigen als Raucher [26]. Dennoch konnte gezeigt werden, dass auch männliche Raucher mit Plattenepithelkarzinomen von Erlotinib profitieren können [27]. In jüngster Zeit konnte gezeigt werden, dass Raucher und Nichtraucher eine unterschiedliche Pharmakokinetik für Erlotinib aufweisen: Sowohl die maximale Plasmakonzentration als auch die Konzentration nach 24 Stunden waren bei Rauchern niedriger als bei Nichtrauchern [28].

Während bisherige Chemotherapiedosierungen in Abhängigkeit von der Körperoberfläche gewählt werden, ist die Initialdosierung von Tyrosinkinasehemmern unabhängig von Größe und 
Körpergewicht. Eine Dosisreduktion ist nur bei relevanten Nebenwirkungen vorgesehen.

Insgesamt fehlen weiterhin Faktoren von prädiktiver und prognostischer Relevanz für die Therapie mit Erlotinib.

In der vorliegenden retrospektiven Analyse von 275 Patienten (233 longitudinal auswertbar), die in der Zweit- und Drittlinie mit Erlotinib behandelt wurden, wurde versucht, die bekannten prognostischen Faktoren weiter zu spezifizieren sowie weitere prognostische Faktoren von hoher klinischer Relevanz herauszuarbeiten.

Neben der Differenzierung des Ausprägungsgrades der Hautnebenwirkungen (Rash Grad 1-4), dem Raucherstatus, Geschlecht und Histologie wurde erstmals der Einfluss von Körpergewicht und Größe und der hieraus ermittelte Body-Mass-Index (BMI) auf das Ansprechen und die Nebenwirkungsrate von Erlotinib untersucht. Daten zum Bezug zwischen Geschlecht, Rash und BMI fehlen bisher gänzlich.

\section{Methoden}

\section{$\nabla$}

Von Oktober 2005 bis Februar 2011 wurden in der Evangelischen Lungenklinik in Berlin-Buch 275 Patienten mit Erlotinib behandelt, von denen 233 Patienten (155 Männer und 78 Frauen) longitudinal auswertbar waren. Es handelte sich um 141 Patienten mit Adenokarzinomen (60,5\%) und 92 Patienten mit Plattenepithelkarzinomen (39,5\%).

175 (75\%) dieser Patienten waren zum Zeitpunkt der Diagnosestellung älter als 60 Jahre.

Die Erlotinib-Dosis lag initial bei allen Patienten bei $150 \mathrm{mg}$ täglich.

Die Patienten wurden in 4-wöchentlichen Abständen in der onkologischen Fachambulanz der Evangelischen Lungenklinik untersucht. Neben der Anamnese, der körperlichen Untersuchung mit Erhebung der Hautnebenwirkungen wurden jeweils Blutbildund Leberwertkontrollen durchgeführt. Nach 8 Wochen wurde eine Einschätzung des Therapieansprechens mittels konventioneller Röntgen-Übersicht und ggfs. Computertomografie des Thorax durchgeführt.

Die Erhebung der Intensität der Hautnebenwirkungen wurde jeweils nach 4 Wochen Einnahme und dann weiter in 4-wöchentlichem Abstand durchgeführt und dokumentiert. Die Einteilung der Intensität wurde immer von demselben Untersucher vorgenommen.

Die Einteilung erfolgte nach der CTCAE (Common Toxicity Criteria for Adverse Events Version 3.0)-Klassifikation in Grad 1 bis 4 : Grad 1: Makulöse oder papulöse Eruption oder Erythem ohne Begleitsymptome

Grad 2: Makulöse oder papulöse Eruption oder Erythem mit Juckreiz oder anderen Begleitsymptomen, lokalisierte Desquamation auf $<50 \%$ der Körperoberfläche

Grad 3: Schwere generalisierte Erythrodermie oder makulöse, papulöse oder vesikuläre Eruption; Desquamation auf $>50 \%$ der Körperoberfläche, einhergehend mit Schmerzen, Ulzeration oder Desquamation

Grad 4: Generalisierte exfoliative, ulzerative oder bullöse Dermatitis.

Für die Therapie des Rash wurde ab Grad 1 steroidhaltige Salbe (Dermatop-Salbe ${ }^{\circledR}$ ) für die betroffenen Partien verordnet. Bei bakterieller Superinfektion wurde Minocyclin $2 \times 50 \mathrm{mg} / \mathrm{die}$ verordnet. Als Basis der Hautpflege wurden stark fetthaltige Cremes empfohlen (Excipial Fettcreme ${ }^{\circledR}$, Linola Fettsalbe ${ }^{\circledR}$ ).
Bei Rash ab Grad 2 wurde Erlotinib für 14 Tage pausiert. Nach Abklingen der Hautveränderungen wurde die Therapie mit der nächstniedrigen Dosis (100 bzw. $50 \mathrm{mg}$ Erlotinib) fortgeführt. Für die überwiegende Anzahl der Patienten wurde die Therapie mit Erlotinib als Zweit- oder Drittlinientherapie nach vorheriger platinbasierter Chemotherapie und Taxanen eingesetzt. Von den 141 Patienten mit Adenokarzinomen erhielten 38 Patienten (26,9 \%) Erlotinib als Zweitlinientherapie, 72 Patienten (51\%) als Drittlinientherapie und 5 Patienten (3,5\%) als Viertlinientherapie.

Bei den Patienten mit Plattenepithelkarzinomen wurden 26 Patienten $(28,3 \%)$ in der Zweitlinie mit Erlotinib behandelt, $52 \mathrm{~Pa}$ tienten $(56,5 \%)$ in der Drittlinie und 2 Patienten $(2,2 \%)$ in der Viertlinie.

132 Patienten (66\%) waren aktive Raucher oder Ex-Raucher, 68 Patienten (34\%) Nichtraucher, von 33 Patienten konnten keine Daten bezüglich des Raucherstatus erhoben werden.

\section{Ergebnisse}

Das Ergebnis bezüglich des Gesamtüberlebens ab dem Zeitpunkt der Erlotinib-Einnahme ist mit den Daten aus der Literatur vergleichbar $(\bullet$ Abb. 1). Die Patienten mit Adenokarzinomen haben ein besseres Overall Survival (OS, median 6,48 Monate) als Patienten mit Plattenepithelkarzinomen (median 6 Monate). In unserem Kollektiv wurde die Indikation für den Einsatz von Erlotinib noch nicht vom EGF-Rezeptor-Mutationsstatus beeinflusst, da diese Methode erst später im Beobachtungszeitraum etabliert wurde. So erklärt sich möglicherweise die geringe Differenz zwischen dem Überleben der verschiedenen histologischen Typen, eine Selektion der Patienten mit Adenokarzinomen hatte vorher nicht stattgefunden.

Ebenfalls mit den Literaturdaten in Übereinstimmung zu bringen sind die Ergebnisse zum Gesamtüberleben nach Beginn der Erlotinib-Einnahme und dem Raucherstatus der Patienten $(\bullet$ Abb.2). Das längste mediane OS mit 8,3 Monaten haben Patienten, die nie geraucht haben. In unserem Patientengut weisen die ehemaligen Raucher das schlechteste mediane Überleben auf

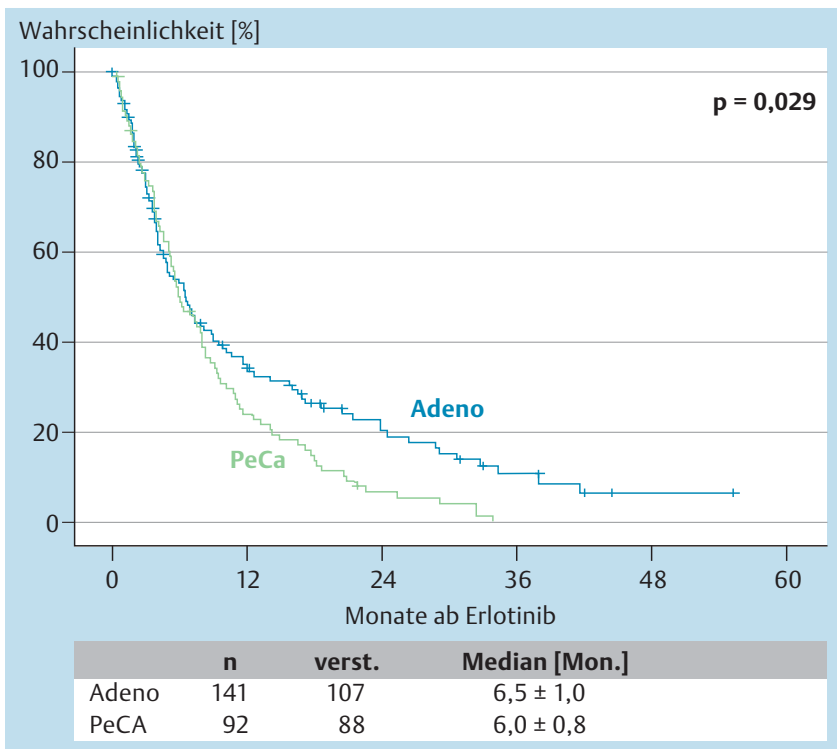

Abb. 1 Overall Survival nach Histologie. Es ergibt sich ein signifikanter Überlebensvorteil für Patienten mit Adenokarzinomen. Adeno=Adenokarzinom, PeCa = Plattenepithelkarziom, $\mathrm{n}=$ Anzahl, verst. = verstorben . 


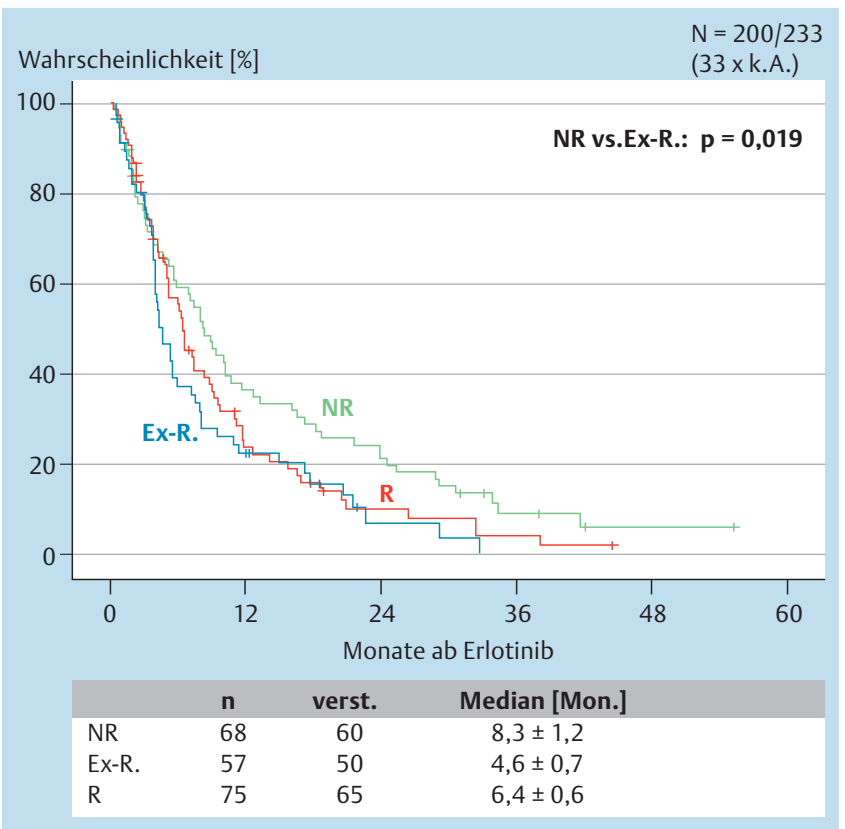

Abb.2 Overall Survival nach Raucherstatus. Lebenslange Nichtraucher haben einen Überlebensvorteil gegenüber Rauchern und Ex-Rauchern unter Erlotinib. NR $=$ Nie-Raucher, Ex-R. $=$ Ex-Raucher, $R=$ Raucher, $n=A n-$ zahl, Verst. $=$ verstorben .

(4,5 Monate), während die aktiven Raucher ein geringfügig besseres medianes Überleben (6,4 Monate) zeigen. Bei fehlender Differenzierung bezüglich der Länge der „Ex-Raucher-Phase“ sind jedoch beide letztgenannten Gruppen in unserer Analyse eher als Einheit zu betrachten.

Von den 233 ausgewerteten Patienten hat kein Patient einen Rash Grad 4 entwickelt ( $\bullet$ Abb.3). Diskrepant zu den Angaben aus der Literatur weisen in unserem Kollektiv die Patienten mit

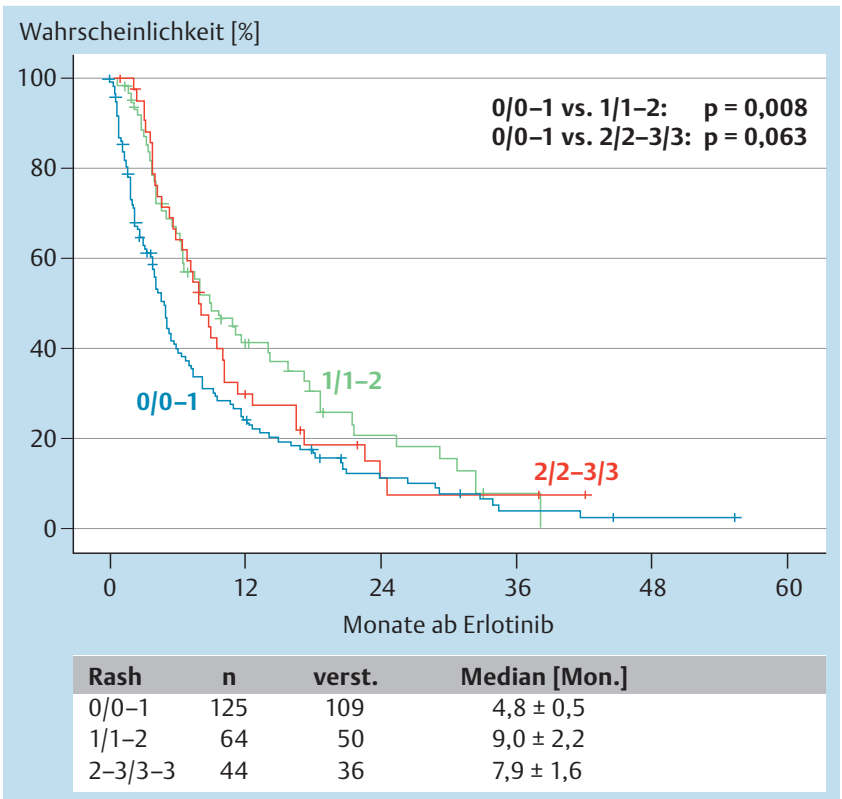

Abb.3 Overall Survival nach Erlotinib-Therapie, nach Rash. Patienten mit mittelgradigem Rash haben die beste Prognose. $\mathrm{n}=$ Anzahl, verst. $=$ verstorben. mittelgradig ausgeprägtem Rash (Grad 1 und 1-2) das beste OS auf (median 9 Monate). Sowohl die Patienten ohne Rash (Grad 0 und $0-1$ ) als auch diejenigen mit starkem Rash (Grad 2, 2-3 und 3) weisen ein schlechteres Gesamtüberleben auf (Grad 0 und 01 median 4,8 Monate, Grad 2, 2-3 und 3 median 7,9 Monate). Reduziert man die Patienten auf die Gruppe, die Erlotinib als Drittlinientherapie erhielt ( $\bullet$ Abb.4), ist diese Tendenz noch klarer zu erkennen ( $\boldsymbol{O}$ Abb.4): Die Patienten mit mittelgradigem Rash (Grad 1 und 1-2) zeigen das beste Gesamtüberleben (median 9,7 Monate), während Patienten ohne Rash und mit stark ausgeprägtem Rash ein schlechteres Gesamtüberleben zeigten (Grad 0 und $0-1: 4,6$ Monate, Grad 2, 2-3 und 3: 7,9 Monate).

Der Raucherstatus beeinflusst offenbar die Wahrscheinlichkeit der Rash-Entwicklung ( $\bullet$ Tab.1). Nur 6,7\% der Raucher entwickeln unter Erlotinib einen Rash Grad 2, 2-3 oder 3, während bei den Nichtrauchern 23,5\% einen Rash Grad 2, 2 - 3 oder 3 entwickeln. Diese Beobachtung könnte die These stützen, dass Tyrosinkinasehemmer bei Rauchern schneller verstoffwechselt werden $[28,29]$.

Die Korrelation des Body-Mass-Index (BMI) mit dem Overall Survival aller Patienten ergibt, dass ein mittlerer bis etwas erhöhter BMI (25 - 30) mit dem besten Gesamtüberleben einhergeht (median 7,4 Monate) ( $\bullet$ Abb.5). Wie zu erwarten, weisen die Patienten mit sehr niedrigem BMI $(<18,5)$ das niedrigste Gesamtüberleben auf (median 4,9 Monate). Allerdings haben die Patienten mit sehr hohem BMI (>30) ein ähnlich schlechtes Gesamtüberleben, was möglicherweise mit der in dieser Gruppe erhöhten Komorbidität (metabolisches Syndrom und die Folgeerkrankungen) zu erklären ist.

Eine Korrelation zwischen niedrigem BMI und starker Ausprägung des Rash unter Erlotinibtherapie konnte nicht hergestellt werden ( $\bullet$ Tab.2): Nur 14,2\% der Patienten mit einem BMI $<18,5$ entwickelten einen Rash Grad 2, 2-3 oder 3, während 19,2\% der Patienten mit einem BMI>30 einen höhergradigen Rash (Grad 2, 2 -3 oder 3) entwickelten. Die Empfehlung einer primären Dosis-

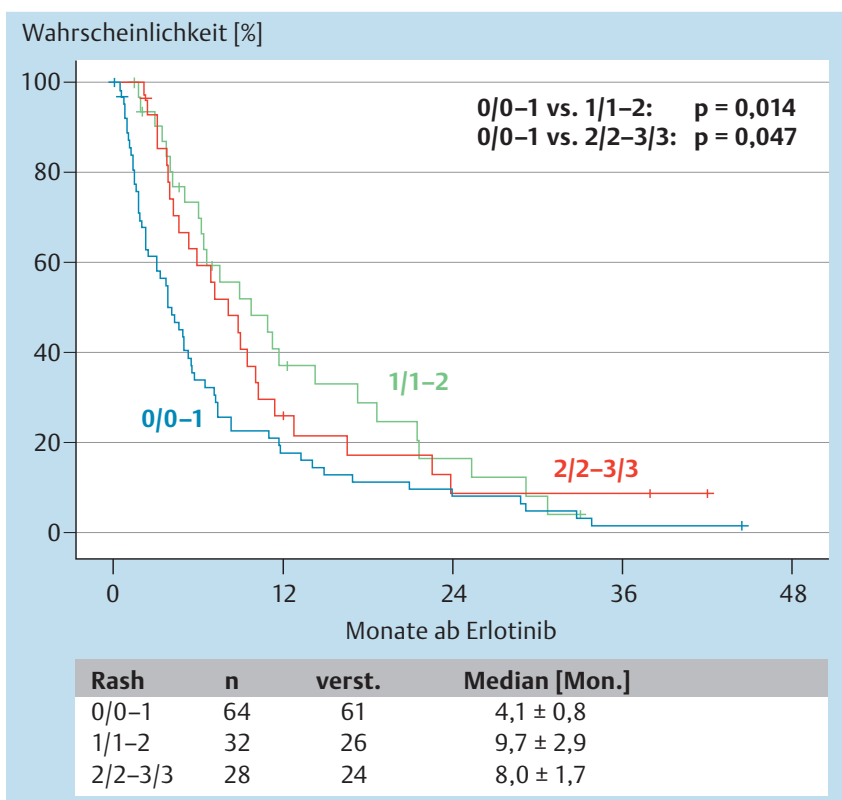

Abb.4 Overall Survival ab Erlotinib-Therapie, nach Rash, nur Drittlinientherapie. Auch bei Reduktion auf die Patienten, die Erlotinib in der dritten Therapielinie erhielten, haben Patienten mit mittelgradigem Rash die beste Prognose. $\mathrm{n}=$ Anzahl, verst. $=$ verstorben . 


\begin{tabular}{|c|c|c|c|c|c|}
\hline \multirow[t]{2}{*}{ Rash } & & \multicolumn{3}{|c|}{ Raucherstatus } & \multirow[t]{2}{*}{ Gesamt } \\
\hline & & Ex & NR & Raucher & \\
\hline \multirow[t]{2}{*}{$0 / 0-1$} & Anzahl & 25 & 37 & 43 & 105 \\
\hline & $\%$ & $43,9 \%$ & $54,4 \%$ & $57,3 \%$ & $52,5 \%$ \\
\hline \multirow[t]{2}{*}{$1 / 1-2$} & Anzahl & 14 & 15 & 27 & 56 \\
\hline & $\%$ & $24,6 \%$ & $22,1 \%$ & $36,0 \%$ & $28,0 \%$ \\
\hline \multirow[t]{2}{*}{$2 / 2-3 / 3$} & Anzahl & 18 & 16 & 5 & 39 \\
\hline & $\%$ & $31,6 \%$ & $23,5 \%$ & $6,7 \%$ & $19,5 \%$ \\
\hline \multirow{2}{*}{ Gesamt } & Anzahl & 57 & 68 & 75 & 200 \\
\hline & $\%$ & $100,0 \%$ & $100,0 \%$ & $100,0 \%$ & $100,0 \%$ \\
\hline
\end{tabular}

Tab. 1 Korrelation zwischen Raucherstatus und Rash $(n=200)$.

$n=$ Anzahl

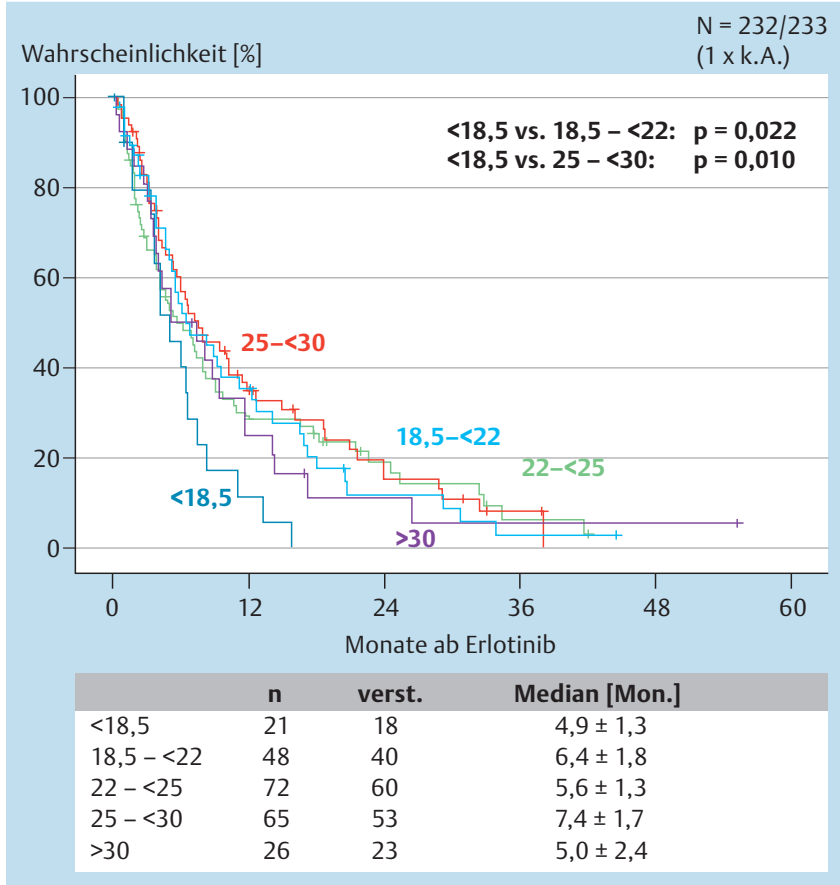

Abb.5 Overall Survival nach Body Mass Index. Patienten mit sehr niedrigem, aber auch mit sehr hohem BMI haben unter Erlotinib die schlechteste Prognose. $n=$ Anzahl, verst. $=$ verstorben .

reduktion bei untergewichtigen Patienten lässt sich aus diesen Daten nicht ableiten.

Insgesamt haben männliche Patienten deutlich häufiger schwerwiegende Hautreaktionen unter Erlotinib entwickelt als weibliche (24,5\% der männlichen Patienten versus 7,8\% der weiblichen Patienten) $(\bullet$ Tab.3 und $\odot$ Tab.4). Bei den Frauen betrug der Anteil an sehr milden Hautreaktionen (Grad 0 und 0-1) 65,9\%, bei den Männern betrug er 48,3\%.

Bei der Korrelation von BMI und Rash ergab sich kein wesentlicher Unterschied zwischen Männern und Frauen.

\section{Diskussion}

Bezüglich der prognostischen Relevanz der Histologie der mit TKI behandelten Patienten mit nichtkleinzelligem Lungenkarzinom stützen die vorliegenden Daten die aus der Literatur bekannten Ergebnisse: Patienten mit Adenokarzinomen (unabhängig vom EGF-Rezeptor-Mutationsstatus) haben unter Erlotinib eine bessere Prognose als Patienten mit Plattenepithelkarzinomen.
Tab. 2 Korrelation zwischen BMI und Rash.

\begin{tabular}{|lcccc|}
\hline BMI-Klassen & \multicolumn{2}{l}{ Rash } & & \\
\cline { 2 - 5 } & $\mathbf{0 / 0 - 1}$ & $\mathbf{1 / 1 - 2}$ & $\mathbf{2 / 2 - 3 / 3}$ & Total \\
\hline$<18,5$ & 13 & 5 & 3 & 21 \\
\hline $18,5-<25$ & 70 & 33 & 17 & 120 \\
\hline $25-<30$ & 28 & 18 & 19 & 65 \\
\hline$>30$ & 14 & 7 & 5 & 26 \\
\hline Total & 125 & 63 & 44 & 232 \\
\hline
\end{tabular}

Die Daten aus der vorliegenden Analyse bezüglich des Rash unter TKI ergänzen die bisher veröffentlichten Ergebnisse [24,30]. Eine Korrelation des Rash zu anderen prognoserelevanten Faktoren, insbesondere beim Lungenkarzinom wie in der vorliegenden Studie, wurde bisher noch nie hergestellt.

Rash wird als prognoserelevanter Faktor der Behandlung mit einem TKI angesehen.

Bei Patienten mit Pankreaskarzinomen wird die starke Ausprägung der Hautreaktion als klarer Indikator für gutes Ansprechen gewertet [32]. Bei fehlender Hautreaktion wird die Therapie oft abgebrochen.

Auch in der vorliegenden Studie haben die Patienten mit sehr wenig oder fehlender Hautreaktion (Grad 0 und $0-1$ ) das schlechteste mediane Überleben, was für einen Zusammenhang der Wirkung auf die Tumorerkrankung mit der Hautreaktion spricht. Der epidermale Wachstumsfaktor (EGF) spielt für die Physiologie von Haut, Schleimhäuten und Haaren eine große Rolle, die genauen Mechanismen und die Ursache für die stark unterschiedliche Ausprägung des Rash bei Patienten unter Erlotinib ist jedoch weiterhin unklar.

In unserem Kollektiv haben die Patienten mit mittelgradigem Rash das beste Gesamtüberleben. Entgegen den Literaturdaten zeigen die Patienten mit stark ausgeprägtem Rash in der vorliegenden Arbeit ein schlechteres Überleben als diejenigen mit mittelgradig ausgeprägtem Rash.

Ursächlich für diese erstmals gemachte Beobachtung könnte eine bei starker Hautreaktion häufig notwendige Dosisreduktion oder Therapiepause sein. Hierdurch kommt es möglicherweise zu einer verringerten Wirksamkeit des Medikaments und somit zu schlechteren Überlebenszeiten der Patienten. In seltenen Fällen zwingt der intensive Rash auch zum Absetzen des TKI nach kurzer Behandlungsdauer, so dass die Behandlungszeit für ein Therapieansprechen nicht ausreicht.

Häufig ist ausgeprägter Rash auch mit starken Diarrhoen vergesellschaftet, was bei vorbestehender Komorbidität auch zu Komplikationen und erhöhter Sterblichkeit führen kann. 


\begin{tabular}{|c|c|c|c|c|c|}
\hline \multirow[t]{2}{*}{ BMI } & & \multicolumn{3}{|l|}{ Rash } & \multirow[t]{2}{*}{ Gesamt } \\
\hline & & $0 / 0-1$ & $1 / 1-2$ & $2 / 2-3 / 3$ & \\
\hline \multirow[t]{2}{*}{$<18,5$} & Anzahl & 6 & 2 & 2 & 10 \\
\hline & $\%$ & $60,00 \%$ & $20,00 \%$ & $20,00 \%$ & $100,00 \%$ \\
\hline \multirow[t]{2}{*}{$18,5-<22$} & Anzahl & 19 & 6 & 5 & 30 \\
\hline & $\%$ & $63,33 \%$ & $20,00 \%$ & $16,67 \%$ & $100,00 \%$ \\
\hline \multirow[t]{2}{*}{$22-<25$} & Anzahl & 23 & 16 & 10 & 49 \\
\hline & $\%$ & $46,94 \%$ & $32,65 \%$ & $20,41 \%$ & $100,00 \%$ \\
\hline \multirow[t]{2}{*}{$25-<30$} & Anzahl & 18 & 13 & 16 & 47 \\
\hline & $\%$ & $38,30 \%$ & $27,66 \%$ & $34,04 \%$ & $100,00 \%$ \\
\hline \multirow[t]{2}{*}{$>30$} & Anzahl & 9 & 5 & 5 & 19 \\
\hline & $\%$ & $47,37 \%$ & $26,32 \%$ & $26,32 \%$ & $100,00 \%$ \\
\hline \multirow[t]{2}{*}{ Gesamt } & Anzahl & 75 & 42 & 38 & 155 \\
\hline & $\%$ & $48,39 \%$ & $27,10 \%$ & $24,52 \%$ & $100,00 \%$ \\
\hline
\end{tabular}

Tab. 3 Korrelation zwischen BMI und Rash bei Männern.

BMI = Body Mass Index

\begin{tabular}{|c|c|c|c|c|c|}
\hline \multirow[t]{2}{*}{ BMI } & & \multicolumn{3}{|l|}{ Rash } & \multirow[t]{2}{*}{ Gesamt } \\
\hline & & $0 / 0-1$ & $1 / 1-2$ & $2 / 2-3 / 3$ & \\
\hline \multirow[t]{2}{*}{$<18,5$} & Anzahl & 7 & 3 & 1 & 11 \\
\hline & $\%$ & $63,64 \%$ & $27,27 \%$ & $9,09 \%$ & $100,00 \%$ \\
\hline \multirow[t]{2}{*}{$18,5-<22$} & Anzahl & 15 & 3 & 0 & 18 \\
\hline & $\%$ & $83,33 \%$ & $16,67 \%$ & $0,00 \%$ & $100,00 \%$ \\
\hline \multirow[t]{2}{*}{$22-<25$} & Anzahl & 13 & 8 & 2 & 23 \\
\hline & $\%$ & $56,52 \%$ & $34,78 \%$ & $8,70 \%$ & $100,00 \%$ \\
\hline \multirow[t]{2}{*}{$25-<30$} & Anzahl & 10 & 5 & 3 & 18 \\
\hline & $\%$ & $55,56 \%$ & $27,78 \%$ & $16,67 \%$ & $100,00 \%$ \\
\hline \multirow[t]{2}{*}{$>30$} & Anzahl & 5 & 2 & 0 & 7 \\
\hline & $\%$ & $71,43 \%$ & $28,57 \%$ & $0,00 \%$ & $100,00 \%$ \\
\hline \multirow[t]{2}{*}{ Gesamt } & Anzahl & 50 & 21 & 6 & 77 \\
\hline & $\%$ & $64,94 \%$ & $27,27 \%$ & $7,79 \%$ & $100,00 \%$ \\
\hline
\end{tabular}

Tab. 4 Korrelation zwischen BMI und Rash bei Frauen.

BMI = Body Mass Index

Die Ausprägung des Rash weist geschlechtsspezifische Unterschiede auf und ist mit einer unterschiedlichen Prognose assoziiert. Die Annahme, dass ausgeprägter Rash (Grad 3) ein günstiges Prognosekriterium ist, gilt insbesondere für Männer nicht. Trotz deutlich häufiger ausgeprägtem Rash haben sie eine schlechtere Prognose als Frauen in der untersuchten Kohorte. Möglicherweise spielt die mangelnde Therapieadhärenz der Patienten, auch aufgrund der oralen Präparation, eine zusätzliche Rolle.

$\mathrm{Zu}$ diskutieren wäre eine unterschiedliche Verstoffwechselung des Erlotinib über die Leber mit resultierenden höheren Plasmaspiegeln bei Männern.

Die Ursache und die klinische Relevanz für den bei Männern häufig stärker ausgeprägten Rash sollte in prospektiven Studien weiter untersucht werden.

Niedrigere Plasmaspiegel für Erlotinib können für aktive Raucher angenommen werden, die in unserem Patientenkollektiv deutlich seltener einen Rash Grad 2, 2-3 oder 3 ausgebildet haben. In einem kleinen Kollektiv von 32 Patienten konnte dies von Hamilton et al. bestätigt werden [28].

Bisher ist eine prospektive Studie mit Messung der Plasmaspiegel von Erlotinib aufgelegt (Current S Studie der Fa. Roche), mit der Zielsetzung, einen Unterschied der Prognose bei Rauchern und Nichtrauchern nachzuweisen und durch Dosisadaption auszugleichen.

Die Bedeutung des Ernährungsstatus für das Überleben von Patienten unter TKI wurde bisher nicht untersucht. Pirlich et al. fanden in einer großen Multicenterstudie, dass in deutschen Krankenhäusern bereits bei Aufnahme 27,4\% der Patienten mangel- ernährt sind. Besonders gefährdet sind Patienten über 60 Jahre, Patienten mit Malignomen sowie Patienten mit Mehrfacherkrankungen [31]. Nicht nur die Lebensqualität, sondern auch die Gesamtprognose der Patienten mit Mangelernährung ist signifikant eingeschränkt, und zwar unabhängig von der Art und Behandlung der malignen Erkrankung [25].

In der vorliegenden Arbeit konnte gezeigt werden, dass der Ernährungsstatus für Patienten mit nichtkleinzelligem Lungenkarzinom unter Therapie mit TKI eine wesentliche Rolle spielt. Mangelernährte Patienten mit einem $\mathrm{BMI}<18$ weisen das schlechteste Gesamtüberleben unter Erlotinib auf. Völlig neu ist jedoch die Erkenntnis, dass Patienten mit starkem Übergewicht $(B M I>30)$ eine fast ebenso schlechte Prognose zeigen wie die unterernährten Patienten. Hierzu existieren bisher keinerlei Daten in der Literatur. Möglicherweise spielt die Komorbidität dieser Patientengruppe mit der resultierenden erhöhten Mortalität eine Rolle. Eventuell ist auch die häufig umfangreiche Komedikation mit Beeinflussung der Resorption des TKI von Bedeutung.

In der vorliegenden Arbeit konnte erstmals gezeigt werden, dass Patienten mit niedrigem BMI nicht häufiger starken Rash entwickeln als normalgewichtige Patienten. Auch ein hoher BMI > 30 führt nicht dazu, dass häufiger stärker ausgeprägte Hautreaktionen auftreten. Diese beiden Patientengruppen weisen gleichzeitig das schlechteste Überleben auf, ohne dass hier ein geschlechtsspezifischer Unterschied zu erkennen wäre. Diese erstmals erstellte Korrelation zwischen den klinischen Faktoren Rash und BMI zeigt, dass beide Faktoren von großer prognosti- 
scher Bedeutung sind. Eine Dosisreduktion kann bei niedrigem BMI nicht primär empfohlen werden.

$\mathrm{Zu}$ diskutieren ist, ob die Begleitmedikation oder die chemotherapeutische Vortherapie der Patienten für die Ausprägung des Rash eine Rolle spielt. Alle unsere Patienten hatten eine platinhaltige Chemotherapie in der Erstlinienbehandlung, kombiniert entweder mit Pemetrexed oder Vinorelbine.

Zusammenfassend lässt sich sagen, dass die bisher nicht untersuchten klinischen Faktoren Rash unter TKI und Ernährungsstatus eine große Rolle für die Prognose der Patienten spielen. Dabei ist der häufig bei Männern intensive Rash mit einer ungünstigeren Prognose verbunden, ein reduzierter und adipöser Ernährungsstatus für Männer und Frauen ebenfalls.

Prospektive Studien zum Verständnis des Rash und der geschlechtsspezifischen Prognoseunterschiede sowie zum Einfluss des Ernährungsstatus sind notwendig.

\section{Interessenkonflikt}

B. Böker hat von der Firma Roche in den letzten fünf Jahren zweimal eine Kongressteilnahme erstattet bekommen, hat zweimal einen Vortrag gegen Honorar gehalten und war einmal an einer Studie beteiligt.

H. Lüders gibt an, dass kein Interessenkonflikt besteht.

C. Grohé hat an Advisory Board Meetings der Firma Roche teilgenommen und Vorträge gehalten, die von der Firma Roche unterstützt wurden. Interessenkonflikte bestehen dennoch nicht.

\section{Literatur}

1 Goeckenjan G, Sitter H, Thomas $M$ et al. Prävention, Diagnostik, Therapie und Nachsorge des Lungenkarzinoms, Interdisziplinäre S3-Leitlinie der Deutschen Gesellschaft für Pneumologie und Beatmungsmedizin und der Deutschen Krebsgesellschaft. Pneumologie 2010; 64 (Supplement 2): S23-S155

2 Jackman DM, Johnson BE. Small-cell lung cancer. Lancet 2005; 366: $1385-1396$

3 Schiller JH, Harrington D, Belani CP et al. Comparison of four chemotherapy regimens for advanced non-small-cell lung cancer. N Engl J Med 2002; 346: $92-98$

4 Pirker J, Pereira JR, Szczesna A et al. Cetuximab plus chemotherapy in patients with advanced non small-cell lung cancer (FLEX); an open label randomised phase III trial. Lancet 2009; 373: 1525 - 1531

5 Scagliotti GV, Parikh P, von Pawel J et al. Phase III study comparing cisplatin plus gemcitabine with cisplatin plus pemetrexed in chemotherapy-naive patients with advanced-stage non-small-cell lung cancer. J Clin Oncol 2008; 26: 3543 - 3551

6 Shepherd FA, Rodrigues Pereira J, Ciuleanu T et al. Erlotinib in previously treated non-small-cell lung cancer. N Engl J Med 2005; 353: 123-132

7 Bezjak A, Tu D, Seymour $L$ et al. Symptom improvement in lung cancer patients treated with erlotinib: quality of life analysis of the National Cancer Institute of Canada Clinical Trials Group Study BR. 21. J Clin Oncol 2006; 24: 3831 - 3837

8 Mok TS, Wu Y, Thongprasert $S$ et al. Gefitinib or carboplatin-paclitaxel in pulmonary adenocarcinoma. N Engl J Med 2009; 361: 947-957

9 Kobayashi $K$, Inoue $A$, Maemondo $M$ et al. First-line gefitinib versus first-line chemotherapy by carboplatin (CBDCA) plus paclitaxel (TXL) in non-small-cell lung cancer (NSCLC) patients with EGFR mutations: A phase III study (002) by North East Japan Gefitinib Study Group. Proc Am Soc Clin Oncol 2009; 27: abstr 8016

10 Tsurutani J, Mitsudomi T, Mori S et al. A phase III, first-line trial of gefitinib versus cisplatin plus docetaxel for patients with advanced or recurrent non-small cell lung cancer (NSCLC) harbouring activating mutation of the epidermal growth factor receptor (EGFR) gene. ECCO/ ESMO 2009; 7: abstr 505
11 Maeda R, Yoshida J, Ishii G et al. Long-term survival and risk factors for recurrence in stage I non-small cell lung cancer patients with tumors up to $3 \mathrm{~cm}$ in maximum dimension. Chest 2010: Online Publikation DOI 10.1378/chest.09 - 3046

12 Brueckl WM, Schoeberl A, Wirtz RM et al. Increased vascular-endothelial growth factor (VEGF) tumor expression and response to epidermal growth factor receptor inhibitor erlotinib in NSCLC. J Thorac Oncol 2008; 3 : $314-316$

13 Gamou S, Hunts J, Harigai H et al. Molecular evidence for the lack of epidermal growth factor receptor gene expression in small cell lung carcinoma cells. Cancer Res 1987; 47: 2668 - 2673

14 Shigematsu H, Lin L, Takahashi T et al. Clinical and biological features associated with epidermal growth factor receptor gene mutations in lung cancers. J Natl Cancer Inst 2005; 97: 339-346

15 Lynch TJ, Bell DW, Sordella $R$ et al. Activating mutations in the epidermal growth factor receptor underlying responsiveness of non-smallcell lung cancer to gefitinib. N Engl J Med 2004; 350: 2129-2139

16 Paez JG, Janne PA, Lee JC et al. EGFR mutations in lung cancer. Correlation with clinical response to gefitinib therapy. Science $2004 ; 304$ : $1497-1500$

17 Kosaka T, Yatabe Y, Endoh $\mathrm{H}$ et al. Mutations of the epidermal growth factor receptor gene in lung cancer: biological and clinical implications. Cancer Res 2004; 64: 8919-8923

18 Marchetti A, Martella C, Felicioni L et al. EGFR mutations in non-smallcell lung cancer: analysis of a large series of cases and development of a rapid and sensitive method for diagnostic screening with potential implications on pharmacologic treatment. J Clin Oncol 2005; 23: 857 865

19 Mitsudomi T, Kosaka T, Endoh H. Mutations of the epidermal growth factor receptor gene predict prolonged survival after gefitinib treatment in patients with non-small cell lung cancer with postoperative recurrence. J Clin Oncol 2005; 23: 2513-2520

20 Tomida S, Koshikawa K, Yatabe Y et al. Gene-expression-based, individualized outcome prediction for surgically treated lung cancer patients. Oncogene 2004; 23: 5360 - 5370

21 Gazdar AF. Activating and resistance mutations of EGFR in non-small cell lung cancer: role in clinical response to EGFR tyrosine kinase inhibitors. Oncogene 2009; 28 (Suppl 1): S24-S31

22 Jackman DM, Miller VA, Cioffredi LA et al. Impact of epidermal growth factor receptor and KRAS mutations on clinical outcomes in previously untreated non-small cell lung cancer patients: results of an online tumor registry of clinical trials. Clin Cancer Res 2009; 15: 5267 - 5273

23 Vamvakas $L$ et al. Pemetrexed compared with erlotinib in pretreated patients with advanced non-small cell lung cancer: Results of a randomized phase III Hellenic Oncology Research group trial. JCO 2010; 28: 7 Abstract 7519

24 Wacker B et al. Correlation between development of rash and efficacy in patients treated with the epidermal growth factor receptor tyrosine kinase inhibitor erlotinib in two large phase III studies. Clin Cancer Res 2007; 13: 3913 - 3921

25 Norman $K$ et al. Cutoff percentiles of bioelectrical phase angle predict functionality, quality of life and mortality in patients with cancer. Am J Clin Nutr 2010; 92: 612-619

26 Tsao AS et al. Smoking affects treatment outcome in patients with advanced nonsmall cell lung cancer. Cancer 2006; 106: 2428-2436

27 Clark GM et al. Clinical benefit of erlotinib in male smokers with squamous-cell NSCLC; ASCO Annual Meeting Proceedings Part I. J Clin Oncol 2006; 24 (Suppl 7166): 18

28 Hamilton $M$, Wolf JL, Rusk J et al. Effects of Smoking on the Pharmakokinetics of Erlotinib. Clin Cancer Res 2006; 12: 2166-2171

29 Renouf DJ, Tang PA, Chen EX et al. An analysis of the effect of smoking status on Study of erlotinib in advanced pancreatic cancer. J Clin Oncol 2010; 28 (Suppl): 4056

30 Mazzoni $F$, Rotella $V$ et al. From clinical trials to clinical practice: predictors of response to erlotinib in advanced non-small cell lung cancer patients pretreated with chemotherapy. Tumori 2011; 97: 160-165

31 Pirlich M, Schütz T, Norman K et al. The German Hospital Malnutrition Study. Clin Nutr 2006; 25: 563-572

32 Adler G, Seufferlein T, Bischoff SC et al. S3-Leitlinie "Exokrines Pankreaskarzinom“. Z Gastroenterol 2007; 45: 487-523 